\section{Rapid modified QuEChERS method for pesticides detection in honey by high-performance liquid chromatography UV-visible}

\author{
Elisabetta Bonerba, Edmondo Ceci, \\ Nicola Montemurro, Giuseppina Tantillo, \\ Angela Di Pinto, Gaetano Vitale Celano, \\ Giancarlo Bozzo
}

Dipartimento di Medicina Veterinaria, Università degli Studi di Bari Aldo Moro, Valenzano (Bari), Italy

\section{Abstract}

The extensive use of pesticides in agriculture plays an important role in bees die-off and allows the presence of residues in hive products, particularly in honey. An accurate and reliable analytical method, based on QuEChERS extractive technique, has been developed for the quantitative determination by high-performance liquid chromatography UV-visible detector of 5 pesticides (Deltamethrin, Dimethoate, Imidacloprid, Acetamiprid, Chlorfenvinphos) in honey. The method, according to Commission Directive 2002/63/EC and Regulation 882/2004/EC, provided excellent results with respect to linearity (correlation coefficient up to 0.993 ), limits of detection and quantification (0.005 and 0.01 $\mu \mathrm{g} / \mathrm{mL}$ for Dimethoate, Deltamethrin and Chlorfenvinphos; 0.02 and $0.05 \mu \mathrm{g} / \mathrm{mL}$ for Acetamiprid and Imidacloprid), recovery values ( 86.4 to $96.3 \%$ ), precision and relative expanded uncertainty of a measurement, demonstrating the conformity of the this method with the European directives. The proposed method was applied to 23 samples of Apulian honey. None of the investigated pesticides was detected in these samples.

\section{Introduzione}

Le api sono insetti molto importanti per l'uomo poiché, oltre a produrre miele, cera, propoli, polline, pappa reale, veleno e loro derivati, hanno un ruolo fondamentale nell'impollinazione dei fiori di molte piante da frutto ed ortaggi (Lautenbach et al., 2012). La capacità impollinatrice delle api è molto elevata se si pensa che ciascun ape visita mediamente 700 fiori al giorno e, tenendo conto che in un alveare ci sono all'incirca 20.000 api bottinatrici, si può facilmente intuire che ogni giorno, con un raggio di circa $3 \mathrm{~km}$ dall'arnia, le api di un alveare sono in grado di impollinare circa 14 milioni di fiori (Kremen et al., 2007). Inoltre, l'ape ha assunto negli anni la rilevante funzione di sentinella biologica poiché interagisce intimamente con l'ambiente circostante in cui vive, prelevando nettare, polline, melata e propoli da fiori e piante, raccogliendo acqua da risorse idriche superficiali e dalla guttazione fogliare ed entrando in contatto con tutte le sostanze aerodisperse. Nell'ultimo decennio si è osservata, a livello internazionale, una diffusa e progressiva crisi nel settore dell'apicoltura dovuta ad estese morie di api che hanno portato, in alcuni casi, al completo spopolamento degli alveari (Kluser e Peduzzi, 2007; Hendricks et al., 2009). Questo fenomeno, indicato come colony collapse disorder (CCD) o sindrome da spopolamento degli alveari (SSA), tra le ipotesi scientifiche più accreditate presenti in bibliografia, può essere ricondotto all'impiego massivo di prodotti fitosanitari in agricoltura (Lebuhn et al., 2013). Il termine fitofarmaci, spesso impropriamente sostituito da pesticidi (dall'inglese pesticides), comprende diverse classi di composti inorganici, organici naturali e di sintesi, formulati commercialmente al fine di combattere, prevenire e/o curare le infezioni causate ai vegetali da organismi nocivi (quali funghi, batteri, insetti, acari, nematodi, virus, micoplasmi e roditori) e contrastare 0 eliminare specie vegetali indesiderate (piante infestanti). Negli ultimi vent'anni il mercato dei prodotti fitosanitari è stato dominato da tre gruppi di composti chimici: organofosfati (agiscono analogamente ad una neurotossina); carbammati (causano un avvelenamento degli inibitori della colinesterasi); piretroidi, (operano sui canali al sodio neuronali) (Smith e Stratton, 1986; WHO, 1989; Ecobichon, 2000). Più recentemente è stata sintetizzata una nuova classe di molecole, i neonicotinoidi, che agiscono come agonisti sui recettori postsinaptici nicotinici dell'acetilcolina (nAChR) (Tomizawa e Casida, 2003) bloccando la normale azione di scambio di ioni attivata dall'acetilcolina. Queste molecole hanno nel tempo registrato un incremento notevole d'uso, sostituendo carbammati, fosforganici e piretroidi, e diventando così la classe di fitofarmaci più diffusa al mondo; il loro impiego è legato all'eccellente efficacia verso numerosi parassiti delle piante, unita ad una relativamente bassa tossicità nei confronti dell'ambiente e soprattutto dei mammiferi, se confrontati con pesticidi di vecchia concezione.

Una corposa legislazione dell'UE disciplina la commercializzazione e l'impiego dei prodotti fitosanitari, e la potenziale presenza di residui di tali prodotti negli alimenti e nei mangimi. I fitofarmaci sono regolamentati principalmente dalla direttiva 91/414/CEE (Commissione Europea, 1991) relativa all'immissione in commercio dei prodotti fitosanitari che non possono essere commercializzati o utilizzati se non precedentemente autorizzati. I limiti mas-
Correspondence: Elisabetta Bonerba, Dipartimento di Medicina Veterinaria, Università degli Studi di Bari Aldo Moro, strada provinciale per Casamassima km 3, 70010 Valenzano (BA), Italy.

Tel. +39.080 .5443850 - Fax: +39.080 .5443855 .

E-mail: elisabetta.bonerba@uniba.it

Key words: Pesticides, Honey, QuEChERS, HPLC UV-Vis, Analytical method.

Received for publication: 10 May 2013.

Revision received: 18 October 2013.

Accepted for publication: 30 October 2013.

This work is licensed under a Creative Commons Attribution 3.0 License (by-nc 3.0).

(C) Copyright E. Bonerba et al., 2014

Licensee PAGEPress, Italy

Italian Journal of Food Safety 2014; 3:1647

doi:10.4081/ijfs.2014.1647

simi ammissibili di fitofarmaci negli alimenti destinati al consumo umano e nei mangimi vengono espressi come livelli massimi di residui (LMR) e sono definiti dal Regolamento (CE) n. 396/2005 del Parlamento Europeo e del Consiglio, concernente i Livelli massimi di residui (LMR) di antiparassitari nei prodotti alimentari e mangimi di origine vegetale e animale, che modifica la precedente direttiva 91/414/CEE e che impone, altresì, a livello comunitario, il monitoraggio e il controllo di tali residui sia negli alimenti di origine vegetale che animale (Commissione Europea, 2005). Questo Regolamento ha subito numerose modifiche ed integrazioni: in particolare il Reg. (CE) n. 178/2006 ha introdotto l'Allegato I recante l'elenco dei prodotti alimentari e dei mangimi cui si applicano i LMR di antiparassitari, e il Reg. (CE) n. 149/2008 ha poi fissato, negli Allegati II, III e IV, i LMR in tutti gli alimenti e mangimi compresi nell'Allegato I (Commissione Europea, 2006, 2008).

Dalla metà del 2003 l'EFSA è responsabile della revisione delle sostanze attive impiegate nei prodotti fitosanitari nei Paesi Membri dell'Unione Europea. Questo compito è assolto dall'unità pesticidi dell'EFSA (PPR), che valuta anche i nuovi principi attivi soggetti ad autorizzazione per l'immissione in commercio, secondo procedure stabilite dalla legislazione e dai più recenti standard e metodi scientifici. A settembre 2008 l'EFSA ha istituito un nuovo Comitato Direttivo, composto da rappresentanti dell'EFSA, della Commissione Europea e degli Stati Membri; a tale organo è stata attribuita la responsabilità di gestire e pianificare la valutazione del rischio cumulativo per i fitofarmaci autorizzati: vengono, quindi, esaminati gruppi di pesticidi, dotati di struttura chimica ed effetti tossici simili, per verificare se il loro impatto sulla salute umana debba essere 
valutato globalmente piuttosto che singolarmente. A dicembre 2008 l'EFSA ha concluso il proprio lavoro di revisione di tutte le molecole impiegate nei prodotti fitosanitari presenti sul mercato dal 1993, onde consentire alla Commissione di istituire un elenco dei principi attivi che sono autorizzati in tutta l'UE; un ulteriore gruppo di sostanze, già sottoposte a valutazione del rischio, da parte degli Stati membri, è stato invece oggetto di revisione nel 2012. Alla luce della elevata tossicità delle classi di fitofarmaci prevalentemente impiegate, e della loro cospicua persistenza nell'ambiente, risulta necessario sviluppare metodologie analitiche che consentano in breve tempo e a basso costo di rilevare la presenza di tali contaminanti negli alimenti di origine vegetale ed animale.

QuEChERS è un acronimo dato dalle parole: veloce (quick), facile (easy), economico (cheap), efficace (effective), rigoroso (rugged) e sicuro (safe); è un approccio analitico che ha semplificato l'analisi dei residui di fitofarmaci soprattutto per gli alimenti di origine vegetale. Il metodo QuEChERS è stato sviluppato negli anni 2001 e 2002 da M. Anastassiades (Anastassiades et al., 2003) che mise a punto questa tecnica per far fronte a complessità, laboriosità, tempi lunghi di esecuzione e notevoli costi degli ordinari metodi di ricerca di pesticidi. Inizialmente, la metodologia è stata sviluppata per l'analisi di farmaci veterinari (antielmintici e tireostatici) nei tessuti animali, ma dopo averne realizzato il grande potenziale per l'estrazione di composti polari e particolari componenti basici, è stata anche estesa con grande successo a matrici di origine vegetale, per analizzare la presenza di residui di antiparassitari. Il nuovo metodo venne presentato nel giugno 2002 all'European Pesticide Residue Workshop a Roma e l'anno successivo venne pubblicato in maniera dettagliata (BSI, 2007; ISO, 1986) diventando, in breve, la metodica di preparazione dei campioni per l'analisi dei residui di fitofarmaci più usata al mondo, con la quale un singolo analista può preparare 8 campioni in 45 minuti con materiali usa e getta del valore di EUR 1-3. L'efficienza laboratoristica viene migliorata, non solo per quanto attiene la riduzione delle tempistiche di lavoro e del risparmio dei materiali di consumo, ma anche per la possibilità di rilevare simultaneamente tutte le classi di fitofarmaci, inserite nella normativa cogente, e presenti in data-base condivisi a livello internazionale. Il metodo originale è stato modificato negli anni per garantire un'efficiente estrazione dei composti pH-dipendenti (ad esempio acido fenossialcanoico), per ridurre al minimo la degradazione di composti pH-sensibili (ad esempio pesticidi labili in condizioni alcaline e acide) e per aumentare il range di pesticidi e di materie prime da analizzare. L'evoluzione analitica del metodo QuEChERS ha consentito di migliorare il recupero di analiti pH-dipendenti attraverso l'introduzione di sali tampone, portando allo sviluppo delle due metodiche ufficiali: AOAC 2007.01 (AOAC, 2007), se è previsto l'impiego di tampone acetato, e EN 15662 (BSI, 2008) che utilizza tampone citrato. La procedura QuEChERS comporta una serie di semplici fasi analitiche di estrazione e cleanup, ed è quindi veloce e facile da eseguire e poco suscettibile di errori; inoltre, l'estratto finale, essendo dissolto in acetonitrile, offre la massima flessibilità nella scelta della tecnica di analisi per la determinazione quantitativa, anche se liquido e gas-cromatografia rappresentano le tecniche di elezione.

Il presente lavoro accoglie la richiesta dell'EFSA, espressa nel report The 2009 European Union Report on Pesticide Residues in Food, pubblicato il 4 aprile 2012 concernente il monitoraggio dei residui di pesticidi negli alimenti condotto nel 2009 (European Food Safety Authority, 2011), che sollecita tutti gli Stati Membri ad implementare programmi di monitoraggio sulla presenza di fitofarmaci in alimenti e mangimi. I dati raccolti sono necessari alla definizione di norme più rigorose che, attraverso la determinazione di stringenti LMR, tutelino il consumatore. La richiesta dell'EFSA è indirizzata anche alla proposizione di metodi innovativi che consentano, in breve tempo e a bassi costi, di ottenere una sicura determinazione analitica. Pertanto, in questo lavoro la metodica QuEChERS, già validata come Metodo Ufficiale per la determinazione di numerosi principi attivi in alimenti di origine vegetale, è stata impiegata per la ricerca di fitofarmaci nel miele, un alimento di origine animale che per la difficile natura compositiva, indotta dalla presenza di numerosi interferen$t i$, richiede nelle metodologie convenzionali tecniche estrattive particolarmente complesse e caratterizzate da molteplici step analitici. Le 5 molecole oggetto della presente indagine appartengono a differenti classi di fitofarmaci: organofosfati (Dimetoato, Clorfenvinfos), piretroidi (Deltametrina), neonicotinoidi (Acetamiprid e Imidacloprid). Formula di struttura, formula chimica e nome IUPAC sono riportati in Figura 1. La determinazione quantitativa è stata condotta mediante cromatografia liquida ad alta prestazione con rivelatore UV-visibile (HPLC UV-Vis), poiché i principi attivi indicati assorbono tutti nell'UV-visibile, anche se a differenti lunghezze d'onda.

\section{Materiali e Metodi}

\section{Campionamento}

Al fine di dimostrare l'applicabilità del metodo di seguito descritto, sono stati prelevati e analizzati 23 campioni di miele millefiori prodotti nelle Province di Lecce e Brindisi tra giu- gno e settembre 2012; le zone di produzione del miele sottoposto ad indagine sono riportate in Tabella 1. I campioni sono stati trasportati presso il laboratorio di Chimica degli Alimenti della Sezione di Sicurezza degli Alimenti e stoccati a temperatura ambiente.

\section{Reattivi}

I reattivi utilizzati sono: $\mathrm{H}_{2} \mathrm{O}$ HPLC-grade (Sigma-Aldrich, St. Louis, M0, USA); Acetonitrile HPLC-grade (Sigma-Aldrich); $\mathrm{Na}_{2} \mathrm{SO}_{4}$ anidro (Sigma-Aldrich); Dimetoato: Dimethoate PESTANAL ${ }^{\circledR}$ (Sigma-Aldrich); Clorfenvinfos: Chlorfenvinphos PESTANAL ${ }^{\circledR}$, mixture of $\mathrm{z}$ - and $\mathrm{e}$ - isomers (Sigma-Aldrich); Deltametrina: Deltamethrin PESTANAL ${ }^{\circledR}$ (Sigma-Aldrich); Acetamiprid: Acetamiprid PESTANAL $^{\circledR}$ (Fluka, St. Louis, M0, USA); Imidacloprid: Imidacloprid PESTANAL ${ }^{\circledR}$ (Supelco, St. Louis, M0, USA).

\section{QuEChERS}

Il metodo QuEChERS comprende: Generon BarNeK $\mathrm{S}=4 \mathrm{~g} \mathrm{MgSO}_{4}, 1 \mathrm{~g} \mathrm{NaCl}, 1 \mathrm{~g}$ TCD, 500 mg DSHS e Generon BarNeK Q=900 mg $\mathrm{MgSO}_{4}, 150 \mathrm{mg}$ primary secondary amine (PSA), $150 \mathrm{mg} \mathrm{C}_{18}$. I BarNek QuEChERS utilizzati contengono differenti reattivi in fase solida, alcuni dei quali coperti da segreto brevettuale, con precise funzioni analitiche necessarie alle fasi di estrazione e clean-up, sono: BarNeK S extraction reagents e BarNeK Q clean-up reagents.

Tra i primi vi sono: MgS04 anidro (facilita la ripartizione dei solventi e migliora il recupero di analiti polari); $\mathrm{NaCl}$ (riduce la quantità degli interferenti polari); TCD e DSHS (regolano il $\mathrm{pH}$ e prevengono la degradazione di analiti sensibili alle variazioni di $\mathrm{pH}$, mantenendo stabile il $\mathrm{pH}$ ). Tra i secondi vengono usati: $\mathrm{C}_{18}$ (rimuove l'interferenza di composti di natura lipofila, quali acidi grassi a lunga catena, steroli e altre sostanze non polari); PSA (rimuove zuccheri, acidi grassi a corta catena, acidi organici, lipidi ed alcuni pigmenti. La combinazione con $\mathrm{C} 18$ rafforza l'efficacia nella rimozione di lipidi e steroli); $\mathrm{MgSO}_{4}$ anidro (rimuove l'eventuale presenza di acqua dalla fase organica).

\section{Strumentazione analitica}

L'analisi cromatografica è stata effettuata mediante cromatografo liquido ad alta efficienza (HPLC) (Agilent Technologies, Santa Clara, CA, USA) modello 1260 Infinity, composto dal modulo delle pompe e dall'iniettore Rheodyne con loop da $20 \mu \mathrm{L}$. La separazione cromatografica è stata eseguita su colonna analitica Synergi Hydro Phenomenex (3 $\mu \mathrm{m}$ $150 \mathrm{~mm}$ x 4,6 mm diametro interno). Come rivelatore è stato utilizzato un detector UV-Vis, isolando le seguenti lunghezze d'onda: $\lambda=200$ $\mathrm{nm}$ (Dimetoato), $\lambda=240 \mathrm{~nm}$ (Clorfenvinfos), $\lambda=225 \quad \mathrm{~nm} \quad$ (Deltametrina), $\lambda=245$ 
(Acetamiprid), $\lambda=270 \mathrm{~nm}$ (Imidacloprid); gestione del segnale ottenuto con software HPLC 2D ChemStation (Agilent Technologies). L'eluizione isocratica è stata effettuata a temperatura ambiente, utilizzando come fase mobile Acqua/Acetonitrile (50:50) ed un flusso di $2,0 \mathrm{~mL} / \mathrm{min}$.

\section{Preparazione del campione}

Per la valutazione del metodo analitico proposto è stato utilizzato, come matrice di riferimento (bianco o controllo negativo), un campione di miele biologico esente da fitofarmaci. Tutti i campioni di miele sono stati dapprima sottoposti a riscaldamento blando $\left(35^{\circ} \mathrm{Cx} 10\right.$ min), onde permetterne la completa fluidificazione e consentire di effettuare una corretta pesata. Dieci g di ciascun campione sono stati esattamente pesati in una falcon $(50 \mathrm{~mL})$ e sono stati addizionati $10 \mathrm{~mL}$ di $\mathrm{H}_{2} \mathrm{O}$ (HPLC grade), secondo quanto previsto dalla metodica estrattiva ufficiale che indica, per alimenti con un contenuto di acqua inferiore all' $80 \%$, una preliminare diluizione, e $3 \mathrm{~g}$ di BarNeK S; successivamente sono stati aggiunti $10 \mathrm{~mL}$ di acetonitrile (HPLC grade). Il tubo è stato agitato vigorosamente per circa 1 min e sottoposto a centrifugazione per 5 minuti a $5000 \mathrm{rpm}$.

Il surnatante recuperato è stato posto in BarNeK Q (15 mL tube), il tubo è stato agitato vigorosamente per circa 1 min e successivamente posto in congelatore a $-20^{\circ} \mathrm{C}$ overnight, al fine di ottimizzare la cristallizzazione di zuccheri interferenti nella fase acquosa, considerando anche la bassa solubilità degli stessi nella fase organica. La fase organica recuperata è stata posta in un tubo da centrifuga e addizionata con $5 \mathrm{~g}$ di $\mathrm{Na}_{2} \mathrm{SO}_{4}$ anidro, al fine di eliminare la possibile presenza della fase acquosa; dopo aver agitato per circa $1 \mathrm{~min}$, il tubo è stato sottoposto nuovamente a centrifugazione per 5 min a $3000 \mathrm{rpm}$. Venti $\mu \mathrm{L}$ del surnatante recuperato sono stati iniettati in HPLC UV-Vis.

\section{Stock solution e work solution}

A partire da ciascuno standard dei cinque fitofarmaci sottoposti a indagine, sono state preparate in matracci tarati $(10 \mathrm{~mL}$, Class $A$ certified) soluzioni madre (stock solution) con una concentrazione di principio attivo pari a $1000 \mu \mathrm{g} / \mathrm{mL}$ in fase mobile (Acqua/Acetonitrile 50:50). Le stock solutions sono state diluite, nuovamente, in fase mobile ottenendo soluzioni di lavoro (work solution) con una concentrazione pari a $10 \mu \mathrm{g} / \mathrm{mL}$. Le stock solution preparate sono state mantenute a $+4^{\circ} \mathrm{C}$ e sono stabili per sei settimane.

\section{Validazione del metodo Linearità}

Lo studio di linearità del metodo è stato effettuato, per tutti i fitofarmaci oggetto di questa indagine, nel range di concentrazione compresa tra $0,01-1,00 \mu \mathrm{g} / \mathrm{mL}$, costruendo curve di calibrazione a cinque punti riferite alla matrice di riferimento, miele esente da fitofarmaci.

\section{$L O D, L O Q$ e $R^{2}$}

La sensibilità del metodo messo a punto è stata valutata attraverso la stima analitica di: LOD [rappresenta il limite inferiore per il quale un segnale analitico è statisticamente differente dai valori del fondo ed è pari a tre volte il rapporto segnale/rumore (S/N)]; LOQ (rappresenta la minima concentrazione rilevabile con un errore accettabile ed è pari a dieci volte il rapporto segnale/rumore); $\mathrm{R}^{2}$ (indica la correlazione esistente tra due serie di dati (concentrazione/area del picco) e serve a stabilire la relazione tra le due proprietà prese in considerazione).

\section{Accuratezza e precisione}

La matrice di riferimento è stata fortificata con due concentrazioni note $(0,5$ e 1,00 $\mu \mathrm{g} / \mathrm{mL}$ ) di ciascun fitofarmaco sottoposto a indagine. Dopo l'aggiunta delle concentrazioni note, è stata effettuata la metodica estrattiva QuEChERS, prima descritta per la preparazione del campione. L'estratto è stato analizzato in HPLC UV-Vis, effettuando tre replicati per ogni concentrazione di fitofarmaco testata. Sono stati quindi valutati recupero percentuale medio, deviazione standard e deviazione standard relativa $\%$.

\section{Incertezza di misura}

L'incertezza di misura è stata determinata per tutte le 5 molecole sottoposte ad indagine considerando per ogni analita due concentrazioni $(0,5$ e $1,00 \mu \mathrm{g} / \mathrm{mL})$ di fortificazione del campione di miele di riferimento, esente da fitofarmaci, seguendo quanto riportato nella Guida EURACHEM/CITAC CG 4 (EURACHEM/ CITAC, 2000).

Preliminarmente sono state definite ed individuate tutte le fonti di incertezza, di seguito descritte.

\section{Incertezza di pesata}

L'incertezza relativa alla pesata degli standard analitici e dei campioni miele è stata calcolata impiegando una distribuzione normale ottenuta attraverso l'applicazione della seguente formula:

$$
U 1=\frac{0.00005}{W i}
$$

dove $W i$ è il peso dell'analita o del campione, $\mathrm{e}$ 0.00005 è il valore di incertezza della bilancia analitica impiegata, al 95\% del livello di confidenza riportato nel relativo certificato di taratura.

\section{Incertezza di linearità di calibrazione}

L'incertezza relativa alla curva di calibrazione è stata calcolata secondo la seguente formula:

$$
U 2=\left(\frac{s}{b_{1}}\right)\left(\left\{\frac{1}{p}\right\}+\left\{\frac{1}{n}\right\}+\left\{\frac{\left(c_{0}-c^{\prime}\right)^{2}}{s_{x x}}\right\}\right)^{\frac{1}{2}}
$$

dove $s$ è la deviazione standard, $b_{1}$ è la pendenza della curva, $\mathrm{p}$ è il numero di misurazioni, $\mathrm{n}$ è il numero dei punti impiegati per la costruzione della curva di calibrazione, $c_{0}$ è la concentrazione dell'analita calcolata dalla curva di calibrazione, c' è la media aritmetica delle concentrazioni di standards utilizzati per la costruzione della curva di calibrazione, e $s_{x x}$ è stato calcolato con la seguente equazione:

$$
s_{x x}=\sum\left(c j-c^{\prime}\right)^{2}
$$

dove $j=1,2, \ldots$, n e cj è la concentrazione di ciascuno standard di calibrazione impiegato nella costruzione della curva di calibrazione.

\section{Incertezza associata alla precisione}

L'errore random associato agli step di estrazione, clean-up e analisi HPLC proposti nel presente lavoro è stato calcolato attraverso la determinazione della Deviazione Standard, la quale a sua volta è stata calcolata attraverso ripetute determinazioni degli analiti ed espressa come ripetibilità. La precisione del metodo è stata calcolata applicando la seguente equazione:

$$
U 3=\frac{s}{(\sqrt{n} \cdot x)}
$$

dove $s$ è la deviazione standard dei risultati ottenuti dalla stima del recupero percentuale, n è il numero di prove analitiche, e x rappresenta il valore medio delle concentrazioni recuperate.

\section{Incertezza di volume}

Il volume delle soluzioni preparate in laboratorio è soggetto a tre fonti di incertezza: calibrazione, ripetibilità e temperatura.

La calibrazione è l'incertezza relativa al volume interno certificato di flask (matracci) e pipette. A titolo esemplificativo si riporta quanto dichiarato dal fornitore dei matracci impiegati, il quale precisa un volume pari a $10.00 \pm 0.02 \mathrm{~mL}(\mathrm{~V} \pm \mathrm{a})$, per misurazioni effettuate alla temperatura di $20^{\circ} \mathrm{C}$. Poiché il valore di incertezza viene indicato senza ulteriori informazioni circa il livello di confidenza o il tipo di distribuzione, nel presente lavoro l'incertezza standard è stata calcolata considerando una distribuzione triangolare secondo la 
seguente equazione:

$$
U 4=\frac{(a / \sqrt{3})}{V}
$$

Allo stesso modo l'incertezza di volume relativo alle pipette utilizzate per la preparazione di soluzioni di lavoro e soluzioni standard è stata calcolata applicando la medesima distribuzione triangolare. I contributi indotti dalle operazioni di diluizione effettuate per ogni livello di concentrazione di analiti testati sono stati prima calcolati separatamente, e successivamente combinati al fine di ottenere il valore di incertezza di volume standard.

La ripetibilità è l'incertezza dovuta dalle variazioni volumetriche per le fasi di riempimento è stata considerata effettuando esperimenti ripetuti.

Poiché le temperature del matraccio e/0 della soluzione da misurare possono differire dalla temperatura dichiarata dal fornitore in fase di taratura $\left(20^{\circ} \mathrm{C}\right)$ e rappresentare una ulteriore fonte di incertezza, ogni misurazione volumetrica è stata effettuata in laboratorio ad una T pari a $20 \pm 2^{\circ} \mathrm{C}$. Inoltre l'incertezza nella misurazione potrebbe derivare anche dal coefficiente del volume di espansione dei solventi impiegati: per acqua e acetonitrile tale effetto può essere considerato trascurabile.

\section{Incertezza composta}

L'incertezza composta U è stata calcolata come di seguito indicato:

$$
\begin{aligned}
& U=C x\left[\left(U 1^{2}+U 2^{2}\right.\right. \\
& \left.\left.+U 3^{2}+U 4^{2}\right)^{1 / 2}\right]
\end{aligned}
$$

dove Cx è la media delle concentrazioni di fitofarmaco testate nei due spiked levels considerati. Ai fini del calcolo dell'incertezza estesa (2U), l'incertezza composta è stata moltiplicata per un fattore di copertura $\mathrm{k}$ pari a 2 , a cui corrisponde un livello di confidenza del 95\%.

\section{Risultati}

La prima fase analitica, per la definizione di linearità, sensibilità e riproducibilità del metodo messo a punto, e per valutare l'effetto matrice, è stata la costruzione delle Curve di Calibrazione. Da ciascuna work solution preparata sono state prelevate quantità note $\mathrm{e}$ addizionate alla matrice di riferimento, miele esente da fitofarmaci, preliminarmente sottoposta al metodo estrattivo QuEChERS descritto, ottenendo le seguenti concentrazioni standard di calibrazione: $0,01 \mu \mathrm{g} / \mathrm{mL}$ (std 1); 0,05 $\mu \mathrm{g} / \mathrm{mL}$ (std 2); 0,10 $\mu \mathrm{g} / \mathrm{mL}$ (std 3); 0,50 $\mu \mathrm{g} / \mathrm{mL}$ (std 4); $1,00 \mu \mathrm{g} / \mathrm{mL}$ (std 5) in fase mobile. Sono stati effettuati tre replicati per ogni con- centrazione testata. I parametri LOD, LOQ e R ${ }^{2}$ riferiti alle cinque curve di calibrazione sono riportati nella Tabella 2 . La precisione e l'accuratezza del metodo sono riportati in Tabella 3. L'incertezza di misura combinata, estesa e l'incertezza di misura estesa relativa $\%$, considerate per i due livelli di fortificazione analizzati $(0,5$ e $1,0 \mu \mathrm{g} / \mathrm{mL})$ sono riportate nelle Tabelle 4 e 5. In nessuno dei 23 campioni di miele sottoposti a indagine sono state rilevate concentrazioni di Dimetoato, Clorfenvinfos; Deltametrina; Acetamiprid ed Imidacloprid, superiori al limite di rilevabilità strumentale del metodo messo a punto. A titolo esemplificativo si riporta il cromatogramma relativo allo standard di calibrazione 3 (concentrazione analiti pari a $0,5 \mu \mathrm{g} / \mathrm{mL}$ ) in Figura 2.

\section{Discussione e Conclusioni}

I parametri analitici del metodo proposto nel presente lavoro, valutati secondo i criteri indicati sopra , consentono di attestare la validità della procedura analitica testata sia in fase di estrazione e clean-up che in fase di determinazione quantitativa. La specificità del metodo è facilmente deducibile mettendo in relazione $\mathrm{i}$ risultati positivi di detezione, ottenuti fortificando, a diversi livelli di concentrazione, il campione di riferimento con gli analiti, con i risultati negativi ottenuti dall'analisi diretta dello stesso campione di riferimento, considerato come controllo negativo. Inoltre non è stata evidenziata alcuna interferenza dovuta

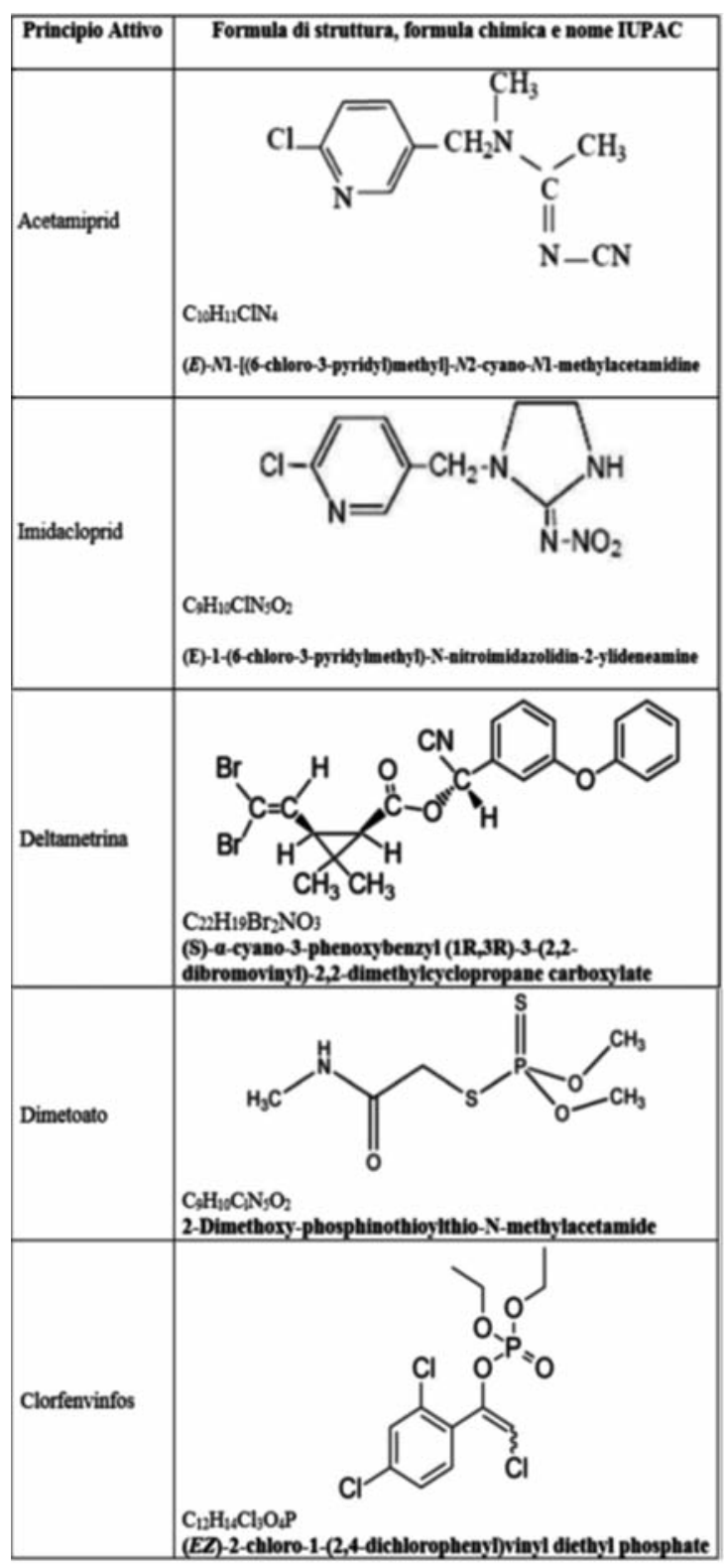

Figura 1. Formula di struttura, formula chimica e nome IUPAC dei 5 fitofarmaci ricercati nel miele. 
Tabella 1. Origine dei campioni di miele sopposti ad indagine.

\begin{tabular}{lccc} 
Campione & Località & Campione & Località \\
1 & Calimera (LE) & 15 & Ceglie Messapica (BR) \\
2 & Copertino (LE) & 16 & San Pietro in Lama (BR) \\
\hline 3 & Cavallino (LE) & 17 & Mesagne (BR) \\
4 & Galatina (LE) & 18 & Brindisi \\
\hline 5 & Galatone (LE) & 19 & Ostuni (BR) \\
6 & Calimera (LE) & 20 & Carovigno (BR) \\
\hline 7 & Calimera (LE) & 21 & Ostuni (BR) \\
8 & Seclì (LE) & 22 & Cisternino (BR) \\
\hline 9 & Lecce & 23 & Ostuni (BR) \\
10 & Racale (LE) & & \\
\hline 11 & Trepuzzi (LE) & & \\
12 & Magliano (LE) & & \\
\hline 13 & Nociglia (LE) & & \\
14 & Borgagne (LE) & & \\
\hline
\end{tabular}

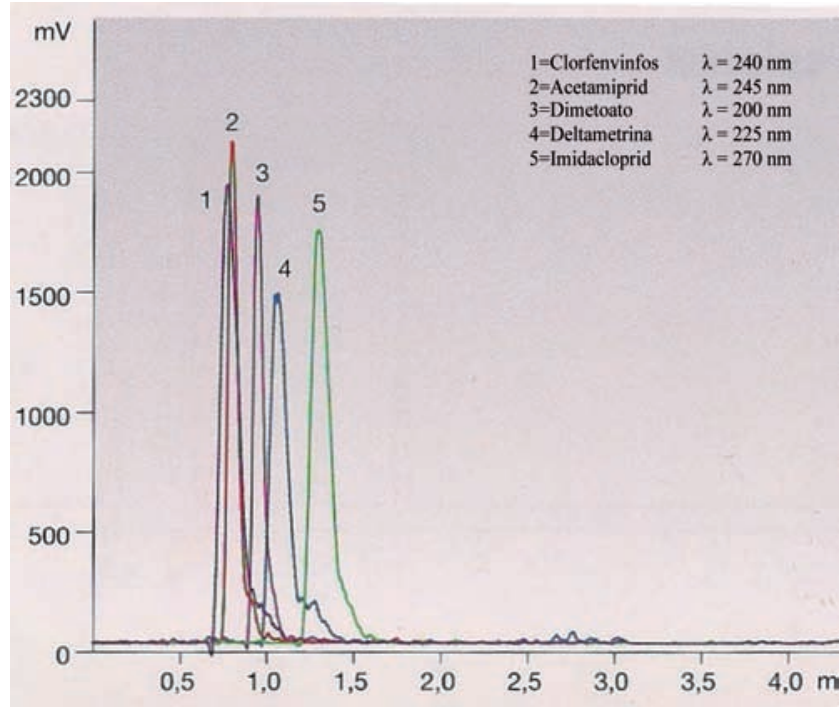

Figura 2. Cromatogramma dei 5 analiti ad una concentrazione pari a $0,5 \mu \mathrm{g} / \mathrm{mL}$.

all'effetto matrice. La linearità delle curve di calibrazione nel range di concentrazione studiato $(0,01-1,00 \mu \mathrm{g} / \mathrm{mL})$ è attestata dai buoni risultati ottenuti per il coefficiente di correlazione $\left(\mathrm{R}^{2}\right) \geq 0,993$. I valori di LOD e LOQ raggiunti consentono di valutare analiticamente la presenza dei 5 fitofarmaci ricercati secondo quanto richiesto e fissato negli LMR stabiliti nella legislazione europea cogente, e riportati in Tabella 6. La semplice procedura estrattiva impiegata, associata alla determinazione HPLC prescelta, ha prodotto soddisfacenti risultati anche per quanto attiene il recupero percentuale, che si attesta nel range $89.2 \%$ (Imidacloprid) - 96.3\% (Clorfenvinfos) per la concentrazione di fortificazione (spiked level) pari a $0,5 \mu \mathrm{g} / \mathrm{mL}$ e $86.4 \%$ (Imidacloprid) 93.2\% (Deltametrina) per la concentrazione di fortificazione (spiked level) pari a $1,0 \mu \mathrm{g} / \mathrm{mL}$. La buona precisione del metodo presentato soddisfa i criteri analitici proposti nel Documento SANCO/12495/2011 (Commissione Europea, 2011), che raccomandano, ai fini della accuratezza e precisione, che il recupero percentuale sia compreso nel range $70-120 \% \mathrm{e}$ il coefficiente di variazione (espresso come Deviazione standard relativa) non superi il $20 \%$. Inoltre nello stesso documento si raccomanda che l'incertezza estesa sia inferiore al $50 \%$.

Considerata la complessità compositiva della matrice alimentare in questione, risulta sorprendente la capacità di estrazione e cleanup della metodica QuEChERS impiegata che, in pochi e facili step analitici, consente la rimozione delle molecole interferenti. Inoltre, pur avendo impiegato un detector UV-Vis, che solitamente restituisce cromatogrammi molto complessi e di difficile interpretazione, la pulizia del campione, iniettato in HPLC, è tale da
Tabella 2. Limit of detection, limit of quantification $\mathbf{e}^{2}$ delle curve di calibrazione.

\begin{tabular}{lccccc}
$\begin{array}{l}\text { Analita } \\
(\mu \mathrm{g} / \mathrm{mL})\end{array}$ & $\begin{array}{c}\text { Range di linearità } \\
(\mu \mathrm{mg} / \mathrm{mL})\end{array}$ & $\begin{array}{c}\text { LOD } \\
(\mu \mathrm{g} / \mathrm{mL})\end{array}$ & LOQ & $\mathbf{R}^{2}$ & $\begin{array}{c}\text { Recupero } \\
(\%)\end{array}$ \\
$\begin{array}{l}\text { Dimetoato } \\
\text { Chlorfenvinphos }\end{array}$ & $0.01-1.0$ & 0.005 & 0.01 & 0.9994 & 94 \\
\hline Deltametrina & $0.01-1.0$ & 0.005 & 0.01 & 0.9996 & 96 \\
Acetamiprid & $0.01-1.0$ & 0.005 & 0.01 & 0.9930 & 96 \\
\hline Imidacloprid & $0.01-1.0$ & 0.02 & 0.05 & 0.9985 & 91 \\
\hline
\end{tabular}

Tabella 3. Recupero, deviazione standard e deviazione standard relativa valutati a differenti concentrazioni di fortificazione (spiked level) della matrice di riferimento.

\begin{tabular}{lcccc} 
Analita & $\begin{array}{c}\text { Recupero } \pm \text { DS }^{\circ} \\
(\%)\end{array}$ & $\begin{array}{c}\text { DSR } \\
(\%)\end{array}$ & $\begin{array}{c}\text { Recupero } \pm \text { DS* } \\
(\%)\end{array}$ & $\begin{array}{c}\text { DSR } \\
(\%)\end{array}$ \\
Dimetoato & $94.1 \pm 2.3$ & 2.44 & $87.9 \pm 2.5$ & 2.84 \\
Clorfenvinfos & $96.3 \pm 2.5$ & 2.59 & $86.5 \pm 1.7$ & 1.96 \\
\hline Deltametrina & $96.2 \pm 2.7$ & 2.80 & $93.2 \pm 2.9$ & 3.11 \\
Acetamiprid & $91.5 \pm 1.6$ & 1.74 & $90.7 \pm 1.4$ & 1.54 \\
\hline Imidacloprid & $89.2 \pm 1.9$ & 2.13 & $86.4 \pm 1.4$ & 1.62 \\
\hline
\end{tabular}

DS, deviazione standard; DSR, deviazione standard relativa. ${ }^{\circ}$ II recupero è stato calcolato come media aritmetica di analisi effettuata in triplicato; lo spiked level $=0,5 \mu \mathrm{g} / \mathrm{mL}$. " Spiked level $=1,0 \mu \mathrm{g} / \mathrm{mL}$

Tabella 4. Incertezza di misura combinata, estesa ed incertezza relativa estesa stimate per la concentrazione di fortificazione della matrice di riferimento pari a $0,5 \mu \mathrm{g} / \mathrm{mL}$.

\begin{tabular}{|c|c|c|c|c|c|c|c|}
\hline & & & rtezza relat & & $\mathrm{U}$ & $2 \mathrm{U}$ & $\mathrm{U} \%$ \\
\hline & Pesata & Curva di calibrazione & Precisione & Volume & & & \\
\hline Dimetoato & 0,00001 & 0,109 & 0,031 & 0,070 & 0,066 & 0,13 & 26,6 \\
\hline Clorfenvinfos & 0,00001 & 0,148 & 0,030 & 0,070 & 0,083 & 0,16 & 33,2 \\
\hline Deltametrina & 0,00001 & 0,129 & 0,029 & 0,070 & 0,074 & 0,14 & 29,9 \\
\hline Acetamiprid & 0,00001 & 0,098 & 0,019 & 0,070 & 0,060 & 0,12 & 24,3 \\
\hline Imidacloprid & 0,00001 & 0,156 & 0,024 & 0,070 & 0,086 & 0,17 & 34,5 \\
\hline
\end{tabular}

$\mathrm{U}$, incertezza di misura combinata; $2 \mathrm{U}$, incertezza di misura estesa; $\mathrm{U} \%$, incertezza relativa estesa. 
ottenere risultati analitici con elevati livelli di risoluzione, buona separazione cromatografica e linea di base stabile. Il metodo descritto, per il quale è richiesto solo un minimo pre-trattamento del campione, rappresenta sicuramente una innovazione analitica nella determinazione dei fitofarmaci nel miele e può essere considerato più veloce delle ormai obsolete tecniche classiche di estrazione. L'impiego nella fase analitica dell'HPLC, uno strumento generalmente presente nei comuni laboratori, anche accademici, definiti small-to-mediumsized, consente di poter superare la problematica relativa agli alti costi richiesti da strumentazioni quali LC o GS-MS, solitamente troppo onerosi per le dimensioni di tali laboratori. L'affidabilità della metodica QuEChERS, già validata per le matrici di origine vegetale, può quindi essere estesa anche ad alimenti di origine animale, come dimostrato dai risultati dei parametri statistici delle curve di calibrazione presentati.

Pertanto, la metodologia analitica proposta può essere considerata un ottimo metodo di screening quantitativo che permette di ottenere dati analitici affidabili sul livello di contaminazione da fitofarmaci nel miele in particolare, e più in generale di comprendere l'impatto ambientale indotto dall'impiego massivo di tali sostanze. L'assenza dei fitofarmaci indagati, nei 23 campioni di miele analizzati, consente inoltre, di proporre alcune considerazioni: i) le zone di allevamento delle api e quelle relative alla raccolta del miele, secondo quanto richiesto dalla legislazione cogente, sono state opportunamente scelte dai diversi produttori, tenendo conto della lontananza da siti di inquinamento correlati ad attività agricole intensive, per le quali l'impiego massivo di fitofarmaci avrebbe potuto indurre la contaminazione dei prodotti apistici; ii) la non rilevazione di principi attivi neonicotinoidi nel miele potrebbe tuttavia confermare l'ipotesi, riportata da numerosi autori, che le api venute in contatto con tali molecole muoiano in così breve tempo, da non riuscire a tornare nell'arnia/nido per la successiva trasformazione e deposizione del miele; perciò, il dato analitico relativo all'assenza di questi fitofarmaci, non è indice assoluto di assenza di contaminazione ambientale; iii) la non rivelazione di principi attivi piretroidi e organofosfati nel miele potrebbe essere altresì dovuta alla totale 0 parziale metabolizzazione 0 eliminazione di tali principi attivi da parte delle stesse api; iv) i fitofarmaci ricercati rappresentano solo una piccola percentuale delle molecole usualmente impiegate nella produzione primaria agricola, di conseguenza non è possibile escludere che altre classi di fitofarmaci, non esplorate nel presente lavoro, possano invece rinvenirsi nel miele.

Avere a disposizione metodi analitici, in grado di fornire, in breve tempo e a bassi costi, risultati quantitativi sulla presenza di residui

Tabella 5. Incertezza di misura combinata, estesa ed incertezza relativa estesa stimate per la concentrazione di fortificazione della matrice di riferimento pari a $1 \mu \mathrm{g} / \mathrm{mL}$.

\begin{tabular}{|c|c|c|c|c|c|c|c|}
\hline & \multicolumn{4}{|c|}{ Incertezza relativa a } & \multirow[t]{2}{*}{$\mathbf{U}$} & \multirow[t]{2}{*}{$2 \mathrm{U}$} & \multirow[t]{2}{*}{$\mathbf{U} \%$} \\
\hline & Pesata & Curva di calibrazione & Precisione & Volume & & & \\
\hline Dimetoato & 0,00001 & 0,020 & 0,035 & 0,070 & 0,080 & 0,16 & 16,1 \\
\hline Clorfenvinfos & 0,00001 & 0,035 & 0,028 & 0,070 & 0,083 & 0,16 & 16,6 \\
\hline Deltametrina & 0,00001 & 0,041 & 0,024 & 0,070 & 0,084 & 0,16 & 16,9 \\
\hline Acetamiprid & 0,00001 & 0,028 & 0,032 & 0,070 & 0,081 & 0,16 & 16,3 \\
\hline Imidacloprid & 0,00001 & 0,051 & 0,037 & 0,070 & 0,094 & 0,18 & 18,8 \\
\hline
\end{tabular}

$\mathrm{U}$, incertezza di misura combinata; $2 \mathrm{U}$, incertezza di misura estesa; $\mathrm{U} \%$, incertezza relativa estesa.

Tabella 6. Livelli massimi di residui dei fitofarmaci analizzati e relativi riferimenti legislativi.

\begin{tabular}{lcl} 
Analita & LMR mg/kg & Riferimento legislativo \\
Dimetoato & LOD & Reg. CE 1097/2009 (Commissione Europea, 2009) \\
Clorfenvinfos & 0,01 & Reg. UE 310/2011 (Commissione Europea, 2011) \\
\hline Deltametrina & 0,01 & Reg. UE 441/2012 (Commissione Europea, 2012) \\
Acetamiprid & 0,05 & Reg. UE 978/2011 (Commissione Europea, 2011) \\
\hline Imidacloprid & 0,05 & Reg. UE 893/2010 (Commissione Europea, 2010) \\
\hline
\end{tabular}

LMR, livelli massimi di residui; LOD, limit of detection.

di xenobiotici negli alimenti destinati al consumo umano, deve rappresentare una priorità nell'attività del medico veterinario ispettore, ai fini della esecuzione di un'attenta analisi del rischio che consenta di attuare opportuni sistemi di prevenzione di filiera.

\section{Bibliografia}

Anastassiades M, Lehotay SJ, Stajnbaher D, Schenck FJ 2003. Fast and easy multiresidue method employing Acetonitile extraction/partitioning and "dispersive solidphase extraction" for the determination of pesticide residues in produce. $\mathrm{J}$ AOAC Int 86:412-31.

AOAC, 2007. AOAC Official Method 2007.01 Pesticide residues in foods by Acetonitrile extraction and partitioning with Magnesium Sulfate gas chromatography/mass spectrometry and liquid chromatography/tandem mass spectrometry. Disponibile al sito: http://www.weber.hu/ PDFs/QuEChERS/AOAC_2007_01.pdf

BSI, 2007. Food analysis. Determination of pesticide residues by LCtMS/MS-tandem mass spectrometric parameters. CEN/TS 15641. British Standards Institution ed., Londra, Gran Bretagna.

BSI, 2008. Foods of plant origin. Determination of pesticide residues using GC-MS and/or LC-MS/MS following acetonitrile extraction/partitioning and clean-up by dispersive SPE-QuEChERS-method. BSI EN 15662:2008. British Standards Institution ed., Londra, Gran Bretagna.

Commissione Europea, 1991. Direttiva del Consiglio del 15 luglio 1991, relativa all'immissione in commercio dei prodotti fitosanitari, 91/414/CEE. In: Gazzetta ufficiale, L 230, 19/08/1991.

Commissione Europea, 2005. Regolamento del Parlamento Europeo e del Consiglio del 23 febbraio 2005 concernente i livelli massimi di residui di antiparassitari nei 0 sui prodotti alimentari e mangimi di origine vegetale e animale e che modifica la direttiva 91/414/CEE del Consiglio, 396/2005/CE. In: Gazzetta ufficiale, L 70/1, 16/03/2005.

Commissione Europea, 2006. Regolamento della Commissione dell'1 febbraio 2006 che modifica il Regolamento (CE) $n$. 396/2005 del Parlamento europeo e del Consiglio per introdurvi l'allegato I, recante l'elenco dei prodotti alimentari e dei mangimi cui si applicano i livelli massimi di residui di antiparassitari, 178/2006/CE. In: Gazzetta ufficiale, L 29/3, 02/02/2006.

Commissione Europea, 2008. Regolamento della Commissione del 29 gennaio 2008 che modifica il Regolamento (CE) n. 396/2005 del Parlamento europeo e del Consiglio e definisce gli allegati II, III e IV, che fissano i livelli massimi di residui per i prodotti compresi nell'allegato I del suddetto regolamento, 149/2008/CE. In: Gazzetta ufficiale, L 58/1, 01/03/2008.

Commissione Europea, 2011. Method validation and quality control procedures for pesticide residues analysis in food and feed. Disponibile al sito: http://ec.europa.eu/food/plant/protection/r esources/qualcontrol_en.pdf

Ecobichon DJ, 2000. Effetti tossici dei pesticidi. In: Kllassen CD, ed. Casarett \& Doull's Tossicologia. I. Fondamenti dell'azione delle sostanze tossiche. Edizioni Mediche 
Scientifiche Internazionali, Roma, pp 847910.

EURACHEM/CITAC, 2000. Quantificazione dell'incertezza nelle misure analitiche. Disponibile al sito: http://www.accredia.it/UploadDocs/1627_Q UAAM2000_1__ITA.pdf

European Food Safety Authority, 2011. The 2009 European union report on pesticide residues in food. EFSA Journal 9:2430.

Hendricks P, Chauzat MP, Debin M, Neuman P, Fries I, Ritter W, Brown M, Mutinelli F, Le Conte Y, Gregorc A, 2009. Bee mortality and bee surveillance in Europe. Disponibile al sito: http:/www.efsa.europa.eu/it/supporting/doc/27e.pdf

ISO, 1986. Precision of test methods. Determination of repeatability and reproducibility for a standard test method by inter-laboratory test. ISO norm 5725:1986.
Organizzazione Internazionale per la Standardizzazione ed., Ginevra, Svizzera.

Kluser S, Peduzzi P, 2007. Global pollinator decline: a literature review. Disponibile al sito: http://www.grid.unep.ch /products/3_Reports/Global_pollinator_decline_literature_review_2007.pdf

Kremen C, Williams NM, Aizen MA, GemmillHerren B, LeBuhn G, Minckley R, Packer L, Potts SG, Roulston Ta, Steffan-Dewenter I, Vazquez DP, Winfree R, Adams L, Crone EE, Greenleaf SS, Keitt TH, Klein A-M, Regetz J, Ricketts TH, 2007. Pollination and other ecosystem services produced by mobile organisms: a conceptual framework for the effects of land-use change. Ecol Lett 10:299-314.

Lautenbach S, Seppelt R, Liebscher J, Dormann CF, 2012. Spatial and temporal trends of global pollination benefit. PLoS
ONE 7:e35954.

Lebuhn G, Droege S, Connor EF, GemmillHerren B, Potts SG, Minckley RL, Griswold T, Jean R, Kula E, Roubik DW, Cane J, Wright KW, Frankie G, Parker F, 2013. Detecting insect pollinator declines on regional and global scales. Conserv Biol 27:113-20.

Smith TM, Stratton GW, 1986. Effects of synthetic pyrethroid insecticides on nontarget organisms. Rev Environ Contam T 97:93117.

Tomizawa M, Casida JE, 2003. Selective toxicity of neonicotinoids attributable to specificity of insect and mammalian nicotinic receptors. Ann Rev Entomol 48:339-64.

WHO, International Programme on Chemical Safety (IPCS) 1989. World Health Organization ed., Ginevra, Svizzera. 